\title{
Transient Solution of a Single Server Queuing Model with Correlated Reneging Using Runge-Kutta Method
}

\author{
Rakesh Kumar \\ School of Mathematics, \\ Shri Mata Vaishno Devi University, \\ Katra, Jammu and Kashmir, Pin-182320, India. \\ Corresponding author: rakesh.kumar@smvdu.ac.in \\ Bhavneet Singh Soodan \\ School of Mathematics, \\ Shri Mata Vaishno Devi University, \\ Katra, Jammu and Kashmir, Pin-182320, India. \\ E-mail: bhavneet5678@gmail.com \\ (Received September 6, 2019; Accepted April 16, 2020)
}

\begin{abstract}
In this paper, the concept of correlated reneging is introduced in queuing theory. The reneging considered so far is dependent on system size, but there are many real life situations where customers may renege due to exogenous factors other than the state of the system. Further, the reneging of customer may induce the other customers to renege at two successive time points. Such reneging is called correlated reneging. An M/M/1/K queuing model with correlated reneging is studied. Runge-Kutta method of fourth order is presented to obtain the transient solution of the model. Some performance measures like expected system size and expected waiting time in the system are studied.
\end{abstract}

Keywords- Queuing model, Correlated reneging, Transient solution, Runge-Kutta method, Transition marks.

\section{Introduction}

Queuing systems with impatient customers are quite useful in the performance analysis of systems like perishable inventory systems, hospitals having critical patients, telephone exchanges and computer communication systems with packet loss. The concept of impatient customers in queuing theory was introduced in the works of Haight (1957, 1959), Ancker Jr and Gafarian (1963a, 1963b), and Rao (1965; 1967). A number of queuing models with reneging and balking were studied since then. In recent past, Kumar and Sharma (2012) put forth a new concept of retention of reneging customers in queuing theory. It was envisaged that if the firms use some customer retention policies then there is a probability that a reneging customer can be retained. Kumar and Sharma (2018, 2019), Sharma et al. (2019) and Sudhesh and Azhagappan (2019) discussed some queuing systems with retention of reneging customers, and performed the transient analyses. Kumar and Soodan (2019) studied the transient behavior of a single server queuing model with correlated arrivals and reneging numerically.

The concept of correlation was first introduced by Mohan (1955) in gambler's ruin problem. Conolly (1968), Conolly and Hadidi (1969), Murari (1969), Mohan and Murrari (1972) studied some queuing models with correlated arrivals. Cidon et al. (1993) considered a queue in which service time is correlated to inter-arrival time with its application in communication systems. Vishneviskii and Dudin (2017) presented the applications of correlated arrival queuing models in telecommunication networks. Fiems and de Turck (2019) investigated a discrete-time queuing 
system with session based arrivals. They evaluated the Taylor-series expansions of the joint probability generating function of the system state at slot boundaries. De Clercq and Walraevens (2020) performed the delay analysis of a priority queuing system having two kinds of arriving customers, viz. external arrivals and the correlated arrivals from other nodes.

In the reneging considered so far, the reneging of a customer was a function of system state. But, this may not fit well in many real life situations where the reneging could be bursty based on exogenous factors, that is, reneging depends on factors other than system state. For example, consider a central system of an online shopping company. The arrival of orders is similar to the arrival of customers, the dispatching of orders is analogous to the service of customers, and the orders cancelled before dispatching (i.e. being served) can be considered as reneging customers. The cancellation of orders (i.e. reneging of customers) could be bursty at times because of the reasons like delay in delivery, some other online shopping companies start offering discounts, getting bad reviews about the products etc. That is, if an order (a customer) is cancelled (reneged) at any time instant, then there is an increased probability that an order may be cancelled at the next time instant. This kind of reneging is referred to as correlated reneging. In the above mentioned example, it is clearly seen that the reneging of one customer induces the other customer to renege without any effect of system state. This motivates us to develop a queuing model with correlated reneging.

In this paper, a finite capacity single server queuing model with correlated reneging is studied. The transient analysis of the model is carried out. The remainder of the paper is arranged as follows: In section 2, the stochastic queuing model is described. In section 3, the mathematical model is presented. Section 4 deals with the transient solution of the model. In section 5, numerical illustration is provided. In section 6, performance measures are given. Finally, the conclusion and future work is provided in section 7.

\section{Stochastic Queuing Model}

We consider a finite capacity single server Markovian queuing model with correlated reneging. The state-transition diagram of the queuing model is shown in Figure 1. The model under investigation is based on the following assumptions:

(i) The customers arrive at a service facility one by one in accordance with Poisson process with parameter $\lambda$.

(ii) There is a single queue and a single server. The service-times are independently, identically and exponentially distributed with parameter $\mu$.

(iii) The capacity of the system is finite (say, $\mathrm{K}$ ), where $\mathrm{K}=\mathrm{N}+1$ and $\mathrm{N}$ is the queue capacity.

(iv) After joining the queue and waiting for some time, a customer may get impatient and leave the queue (renege) without getting service. The reneging of customers can take place only at the transition marks $t_{0}, t_{1}, t_{2}, \ldots$ where $\theta_{r}=t_{r}-t_{r-1}, r=1,2,3 \ldots$, are random variables with $P\left[\theta_{r} \leq x\right]=1-\exp (-\xi x) ; \xi \geq 0, r=1,2,3, \ldots$ i.e. the distribution of inter-transition marks is negative exponential with parameter $\xi$.

(v) The reneging at two consecutive transition marks is governed by the following transition probability matrix: 
International Journal of Mathematical, Engineering and Management Sciences

Vol. 5, No. 5, 886-896, 2020

https://doi.org/10.33889/IJMEMS.2020.5.5.068

to $t_{r}$

$0 \quad 1$

from $t_{r-1} \quad$\begin{tabular}{ll|l}
0 & $p_{00}$ & $p_{01}$ \\
$p_{10}$ & $p_{11}$
\end{tabular}$\|$, where $p_{00}+p_{01}=1$ and $p_{10}+p_{11}=1$.

0 refers to no reneging and 1 refers to the occurrence of reneging. Thus, the reneging at two consecutive transition marks is correlated.

\section{Mathematical Model}

Let us define the following probabilities as:

$Q_{0, r}(t)=$ Probability that at time $\mathrm{t}$ the queue length is zero, the server is idle, and $\mathrm{r}$ is an indicator whether a customer has reneged or not in previous transition mark ( $\mathrm{r}=0$ refers to no reneging and $\mathrm{r}=1$ refers to the occurrence of reneging at previous transition mark).

$P_{0, r}(t)=$ Probability that at time $\mathrm{t}$ the queue length is zero, the server is not idle, and $\mathrm{r}$ is an indicator whether a customer has reneged or not in previous transition mark ( $\mathrm{r}=0$ refers to no reneging and $r=1$ refers to the occurrence of reneging at previous transition mark).

$P_{n, r}(t)=$ Probability that at time $\mathrm{t}$ the queue length is $n(1 \leq n \leq N)$, the server is not idle, and $\mathrm{r}$ is an indicator whether a customer has reneged or not in previous transition mark ( $\mathrm{r}=0$ refers to no reneging and $r=1$ refers to the occurrence of reneging at previous transition mark).

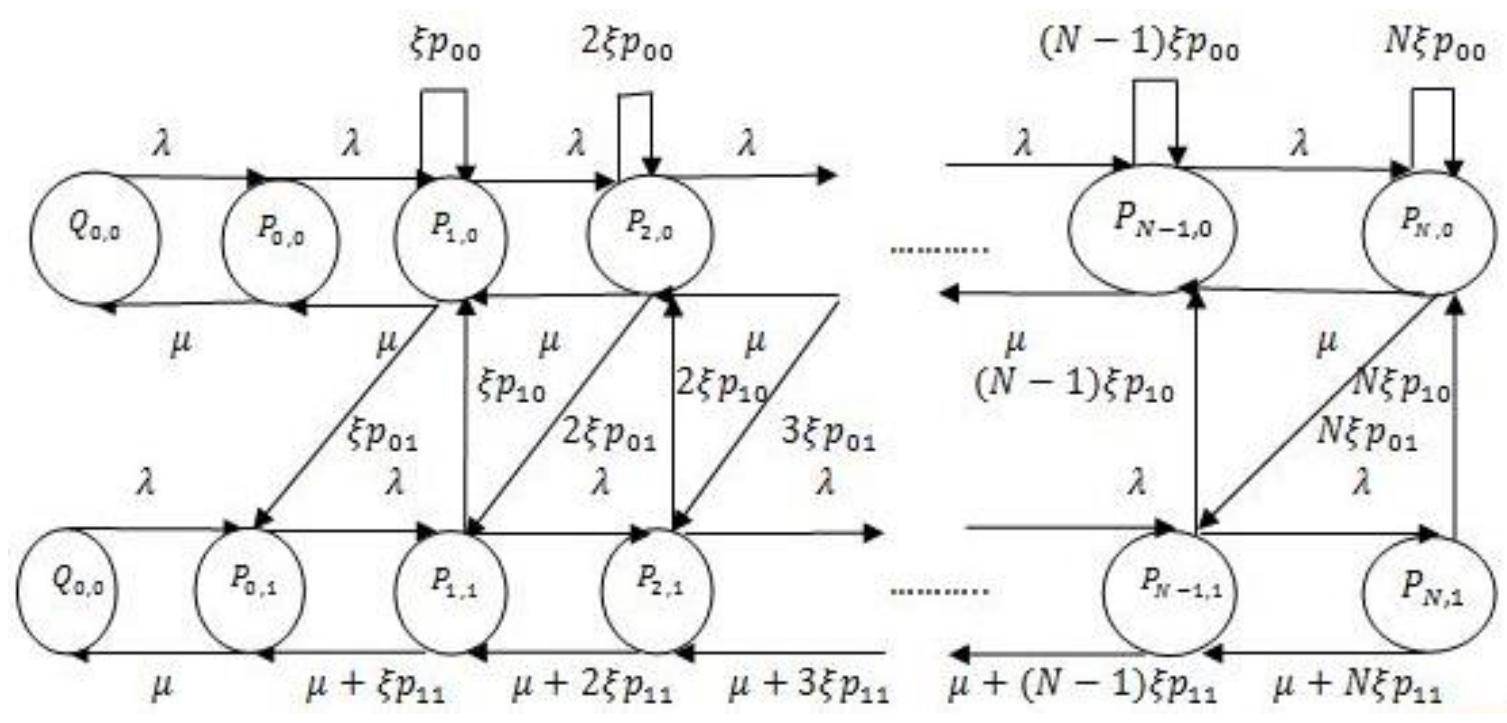

Figure 1: State transition diagram of the queuing model 
The differential equations of the model are:

$$
\begin{aligned}
\frac{d}{d t} Q_{0,0}(t)= & -\lambda Q_{0,0}(t)+\mu P_{0,0}(t) \\
\frac{d}{d t} Q_{0,1}(t)= & -\lambda Q_{0,1}(t)+\mu P_{0,1}(t) \\
\frac{d}{d t} P_{0,0}(t)= & -(\lambda+\mu) P_{0,0}(t)+\mu P_{1,0}(t)+\lambda Q_{0,0}(t) \\
\frac{d}{d t} P_{0,1}(t)= & -(\lambda+\mu) P_{0,1}(t)+\mu P_{1,1}(t)+\lambda Q_{0,1}(t)+\xi\left[p_{11} P_{1,1}(t)+p_{01} P_{1,0}(t)\right] \\
\frac{d}{d t} P_{n, 0}(t)= & -(\lambda+\mu+n \xi) P_{n, 0}(t)+\mu P_{n+1,0}(t)+\lambda P_{n-1,0}(t) \\
& +n \xi\left[p_{00} P_{n, 0}(t)+p_{10} P_{n, 1}(t)\right], 1 \leq n<N \\
\frac{d}{d t} P_{n, 1}(t)= & -(\lambda+\mu+n \xi) P_{n, 1}(t)+\mu P_{n+1,1}(t)+\lambda P_{n-1,1}(t) \\
& +(n+1) \xi\left[p_{01} P_{n+1,0}(t)+p_{11} P_{n+1,1}(t)\right], 1 \leq n<N \\
\frac{d}{d t} P_{N, 0}(t)= & -(\mu+N \xi) P_{N, 0}(t)+\lambda P_{N-1,0}(t)+N \xi\left[p_{00} P_{N, 0}(t)+p_{10} P_{N, 1}(t)\right] \\
\frac{d}{d t} P_{N, 1}(t)= & -(\mu+N \xi) P_{N, 1}(t)+\lambda P_{N-1,1}(t)
\end{aligned}
$$

The initial condition is $P_{0,0}(0)=1$.

\section{Transient Solution of the Model}

Since the transient solution of the model is quite complex to obtain analytically, therefore, we apply the Runge-Kutta Method of fourth-order to obtain the transient solution. In Runge-Kutta method, for solving the differential equation $\frac{d y}{d t}=f(t, y), y\left(t_{0}\right)=y_{0}$. The following formula $y_{i+1}=y_{i}+\left[\frac{k_{1}+2 k_{2}+2 k_{3}+k_{4}}{6}\right] h$, where $h$ is the step size and $k_{1}=h f\left(t_{i}, y_{i}\right), k_{2}=$ $h f\left(t_{i}+\frac{h}{2}, y_{i}+\frac{1}{2} k_{1}\right), k_{3}=h f\left(t_{i}+\frac{h}{2}, y_{i}+\frac{1}{2} k_{2}\right), k_{4}=h f\left(t_{i}+h, y_{i}+k_{3}\right) \quad$ computes $\quad$ an approximate solution.

Let $y=\left\{\begin{array}{c}Q_{0, k}(t) \text { for } n=0, k=0,1 \\ P_{n-1, k}(t) n=1,2,3, \ldots N+1, k=0,1\end{array}\right.$

and $\left\{\begin{array}{c}\frac{d Q_{0, k}(t)}{d t} \quad \text { for } n=0, k=0,1 \\ \frac{d P_{n-1, k}(t)}{d t} \text { for } n=1,2,3, \ldots N+1, k=0,1\end{array}\right.$ corresponds to $f(t, y)$. Therefore, the fourthorder Runge-Kutta method can be applied to solve the differential-difference equations of our model as given below:

Consider a vector PA such that $\boldsymbol{P} \boldsymbol{A}_{n, k}(t)=\left\{\begin{array}{c}Q_{0, k}(t) \quad \text { for } n=0, k=0,1 \\ P_{n-1, k}(t) \text { for } n=1,2,3, \ldots N+1, k=0,1\end{array}\right.$. 
International Journal of Mathematical, Engineering and Management Sciences

Vol. 5, No. 5, 886-896, 2020

https://doi.org/10.33889/IJMEMS.2020.5.5.068

Similarly the vectors $\boldsymbol{A} \boldsymbol{A}_{n, k}, \boldsymbol{A} \boldsymbol{B}_{n, k}, \boldsymbol{A} \boldsymbol{C}_{n, k}$ and $\boldsymbol{A} \boldsymbol{D}_{n, k}$ represent the multivariate version of the intermediate quantities. Vector $\mathbf{A A}$ corresponds to quantity $k_{1}$, therefore,

$$
\begin{aligned}
& \boldsymbol{A} \boldsymbol{A}_{n, k}=(\Delta t) \frac{d \boldsymbol{P} \boldsymbol{A}_{n, k}(t)}{d t}=\left[-\lambda \boldsymbol{P} \boldsymbol{A}_{0,0}(t)+\mu \boldsymbol{P} \boldsymbol{A}_{1,0}(t)\right] \Delta t ; n=0, k=0 \\
& \text { or } \\
& =\left[-\lambda \boldsymbol{P} \boldsymbol{A}_{0,1}(t)+\mu \boldsymbol{P} \boldsymbol{A}_{1,1}(t)\right] \Delta t ; n=0, k=1 \\
& \text { or } \\
& =\left[-(\lambda+\mu) \boldsymbol{P} \boldsymbol{A}_{1,0}(t)+\mu \boldsymbol{P} \boldsymbol{A}_{2,0}(t)+\lambda \boldsymbol{P} \boldsymbol{A}_{0,0}(t)\right] \Delta t ; n=1, k=0 \\
& =\left[-(\lambda+\mu) \boldsymbol{P} \boldsymbol{A}_{1,1}(t)+\mu \boldsymbol{P} \boldsymbol{A}_{2,1}(t)+\lambda \boldsymbol{P} \boldsymbol{A}_{0,1}(t)+\xi\left[p_{11} \boldsymbol{P} \boldsymbol{A}_{2,1}(t)+p_{01} \boldsymbol{P} \boldsymbol{A}_{2,0}(t)\right]\right] \Delta t ; \\
& n=1, k=1 \\
& =\left[-(\lambda+\mu+n \xi) \boldsymbol{P} \boldsymbol{A}_{n+1,0}(t)+\mu \boldsymbol{P} \boldsymbol{A}_{n+2,0}(t)+\lambda \boldsymbol{P} \boldsymbol{A}_{n, 0}(t)\right. \\
& \left.+n \xi\left[p_{00} \boldsymbol{P} \boldsymbol{A}_{n+1,0}(t)+p_{10} \boldsymbol{P} \boldsymbol{A}_{n+1,1}(t)\right]\right] \Delta t ; 1<n \leq N, k=0 \\
& =\left[-(\lambda+\mu+n \xi) \boldsymbol{P} \boldsymbol{A}_{n+1,1}(t)+\mu \boldsymbol{P} \boldsymbol{A}_{n+2,1}(t)+\lambda \boldsymbol{P} \boldsymbol{A}_{n, 1}(t)\right. \\
& \left.+(n+1) \xi\left[p_{01} \boldsymbol{P} \boldsymbol{A}_{n+2,0}(t)+p_{11} \boldsymbol{P} \boldsymbol{A}_{n+2,1}(t)\right]\right] \Delta t ; 1<n \leq N, k=1 \\
& =\left[-(\mu+N \xi) \boldsymbol{P} \boldsymbol{A}_{N+1,0}(t)+\lambda \boldsymbol{P} \boldsymbol{A}_{N, 0}(t)+N \xi\left[p_{00} \boldsymbol{P} \boldsymbol{A}_{N+1,0}(t)+p_{10} \boldsymbol{P} \boldsymbol{A}_{N+1,1}(t)\right]\right] \Delta t ; \\
& \mathrm{n}=N+1, k=0
\end{aligned}
$$

or

$$
=\left[-(\mu+N \xi) \boldsymbol{P} \boldsymbol{A}_{N+1,1}(t)+\lambda \boldsymbol{P} \boldsymbol{A}_{N, 1}(t)\right] \Delta t ; n=N+1, k=1
$$

Vector PB corresponds to quantity $\left(y+\frac{1}{2} k_{1}\right)$ such that $\boldsymbol{P B}_{n, k}\left(t+\frac{1}{2} \Delta t\right)=P A_{n, k}(t)+\frac{1}{2} A A_{n, k}$.

Vector $\mathbf{A B}$ corresponds to quantity $k_{2}$, therefore,

$$
\begin{aligned}
& \boldsymbol{A B}_{n, k}=(\Delta t) \frac{d \boldsymbol{P} \boldsymbol{B}_{n, k}\left(t+\frac{1}{2} \Delta t\right)}{d t}=\left[-\lambda \boldsymbol{P} \boldsymbol{B}_{0,0}\left(t+\frac{1}{2} \Delta t\right)+\mu \boldsymbol{P} \boldsymbol{B}_{1,0}\left(t+\frac{1}{2} \Delta t\right)\right] \Delta t \\
& n=0, k=0
\end{aligned}
$$

or

$$
=\left[-\lambda \boldsymbol{P} \boldsymbol{B}_{0,1}\left(t+\frac{1}{2} \Delta t\right)+\mu \boldsymbol{P} \boldsymbol{B}_{1,1}\left(t+\frac{1}{2} \Delta t\right)\right] \Delta t ; n=0, k=1
$$

or

$$
\begin{array}{r}
=\left[-(\lambda+\mu) \boldsymbol{P} \boldsymbol{B}_{1,0}\left(t+\frac{1}{2} \Delta t\right)+\mu \boldsymbol{P} \boldsymbol{B}_{2,0}\left(t+\frac{1}{2} \Delta t\right)+\lambda \boldsymbol{P} \boldsymbol{B}_{0,0}\left(t+\frac{1}{2} \Delta t\right)\right] \Delta t ; \\
n=1, k=0
\end{array}
$$

or

$$
\begin{aligned}
= & {\left[-(\lambda+\mu) \boldsymbol{P B}_{1,1}\left(t+\frac{1}{2} \Delta t\right)+\mu \boldsymbol{P B}_{2,1}\left(t+\frac{1}{2} \Delta t\right)+\lambda \boldsymbol{P} \boldsymbol{B}_{0,1}\left(t+\frac{1}{2} \Delta t\right)+\right.} \\
& \left.\xi\left[p_{11} \boldsymbol{P} \boldsymbol{B}_{2,1}\left(t+\frac{1}{2} \Delta t\right)+p_{01} \boldsymbol{P} \boldsymbol{B}_{2,0}\left(t+\frac{1}{2} \Delta t\right)\right]\right] \Delta t ; n=1, k=1
\end{aligned}
$$

or 
International Journal of Mathematical, Engineering and Management Sciences

Vol. 5, No. 5, 886-896, 2020

https://doi.org/10.33889/IJMEMS.2020.5.5.068

$$
\begin{aligned}
&= {\left[-(\lambda+\mu+n \xi) \boldsymbol{P} \boldsymbol{B}_{n+1,0}\left(t+\frac{1}{2} \Delta t\right)+\mu \boldsymbol{P} \boldsymbol{B}_{n+2,0}\left(t+\frac{1}{2} \Delta t\right)+\lambda \boldsymbol{P} \boldsymbol{B}_{n, 0}(t+\right.} \\
&\left.\left.\frac{1}{2} \Delta t\right)+n \xi\left[p_{00} \boldsymbol{P} \boldsymbol{B}_{n+1,0}\left(t+\frac{1}{2} \Delta t\right)+p_{10} \boldsymbol{P B}_{n+1,1}\left(t+\frac{1}{2} \Delta t\right)\right]\right] \Delta t ; \\
& 1<n \leq N, k=0
\end{aligned}
$$

or

$$
\begin{array}{r}
=\left[-(\lambda+\mu+n \xi) \boldsymbol{P} \boldsymbol{B}_{n+1,1}\left(t+\frac{1}{2} \Delta t\right)+\mu \boldsymbol{P} \boldsymbol{B}_{n+2,1}\left(t+\frac{1}{2} \Delta t\right)+\lambda \boldsymbol{P} \boldsymbol{B}_{n, 1}(t+\right. \\
\left.\left.\frac{1}{2} \Delta t\right)+(n+1) \xi\left[p_{01} \boldsymbol{P} \boldsymbol{B}_{n+2,0}\left(t+\frac{1}{2} \Delta t\right)+p_{11} \boldsymbol{P} \boldsymbol{B}_{n+2,1}\left(t+\frac{1}{2} \Delta t\right)\right]\right] \\
1<n \leq N, k=1
\end{array}
$$

or

$$
\begin{aligned}
= & {\left[-(\mu+N \xi) \boldsymbol{P} \boldsymbol{B}_{N+1,0}\left(t+\frac{1}{2} \Delta t\right)+\lambda \boldsymbol{P} \boldsymbol{B}_{N, 0}\left(t+\frac{1}{2} \Delta t\right)+N \xi\left[p_{00} \boldsymbol{P} \boldsymbol{B}_{N+1,0}(t+\right.\right.} \\
& \left.\left.\left.\frac{1}{2} \Delta t\right)+p_{10} \boldsymbol{P} \boldsymbol{B}_{N+1,1}\left(t+\frac{1}{2} \Delta t\right)\right]\right] \Delta t ; n=N+1, k=0
\end{aligned}
$$

or

$$
=\left[-(\mu+N \xi) \boldsymbol{P} \boldsymbol{B}_{N+1,1}\left(t+\frac{1}{2} \Delta t\right)+\lambda \boldsymbol{P} \boldsymbol{B}_{N, 1}\left(t+\frac{1}{2} \Delta t\right)\right] \Delta t ; n=N+1, k=1 .
$$

Vector $\mathbf{P C}$ corresponds to quantity $\left(y+\frac{1}{2} k_{2}\right)$ such that $\boldsymbol{P} \boldsymbol{C}_{n, k}\left(t+\frac{1}{2} \Delta t\right)=\boldsymbol{P} \boldsymbol{A}_{n, k}(t)+\frac{1}{2} \boldsymbol{A} \boldsymbol{B}_{n, k}$.

Vector $\mathbf{A B}$ corresponds to quantity $k_{3}$, therefore,

$$
\begin{aligned}
& \boldsymbol{A C}_{n, k}=(\Delta t) \frac{d \boldsymbol{P} \boldsymbol{C}_{n, k}\left(t+\frac{1}{2} \Delta t\right)}{d t} \\
&=\left[-\lambda \boldsymbol{P} \boldsymbol{C}_{0,0}\left(t+\frac{1}{2} \Delta t\right)+\mu \boldsymbol{P} \boldsymbol{C}_{1,0}\left(t+\frac{1}{2} \Delta t\right)\right] \Delta t ; n=0, k=0 \\
& \text { or } \quad {\left[-\lambda \boldsymbol{P} \boldsymbol{C}_{0,1}\left(t+\frac{1}{2} \Delta t\right)+\mu \boldsymbol{P} \boldsymbol{C}_{1,1}\left(t+\frac{1}{2} \Delta t\right)\right] \Delta t ; n=0, k=1 } \\
& \text { or } \quad {\left[-(\lambda+\mu) \boldsymbol{P} \boldsymbol{C}_{1,0}\left(t+\frac{1}{2} \Delta t\right)+\mu \boldsymbol{P} \boldsymbol{C}_{2,0}\left(t+\frac{1}{2} \Delta t\right)+\lambda \boldsymbol{P} \boldsymbol{C}_{0,0}\left(t+\frac{1}{2} \Delta t\right)\right] \Delta t ; } \\
& n=1, k=0
\end{aligned}
$$

or

$$
\begin{gathered}
=\left[-(\lambda+\mu) \boldsymbol{P} \boldsymbol{C}_{1,1}\left(t+\frac{1}{2} \Delta t\right)+\mu \boldsymbol{P} \boldsymbol{C}_{2,1}\left(t+\frac{1}{2} \Delta t\right)+\lambda \boldsymbol{P} \boldsymbol{C}_{0,1}\left(t+\frac{1}{2} \Delta t\right)+\right. \\
\left.\xi\left[p_{11} \boldsymbol{P} \boldsymbol{C}_{2,1}\left(t+\frac{1}{2} \Delta t\right)+p_{01} \boldsymbol{P \boldsymbol { C } _ { 2 , 0 }}\left(t+\frac{1}{2} \Delta t\right)\right]\right] \Delta t ; n=1, k=1
\end{gathered}
$$

or

$$
=\left[-(\lambda+\mu+n \xi) \boldsymbol{P} \boldsymbol{C}_{n+1,0}\left(t+\frac{1}{2} \Delta t\right)+\mu \boldsymbol{P} \boldsymbol{C}_{n+2,0}\left(t+\frac{1}{2} \Delta t\right)+\lambda \boldsymbol{P} \boldsymbol{C}_{n, 0}\left(t+\frac{1}{2} \Delta t\right)+\right.
$$

$\left.n \xi\left[p_{00} \boldsymbol{P} \boldsymbol{C}_{n+1,0}\left(t+\frac{1}{2} \Delta t\right)+p_{10} \boldsymbol{P} \boldsymbol{C}_{n+1,1}\left(t+\frac{1}{2} \Delta t\right)\right]\right] \Delta t ; 1<n \leq N, k=0$

or

$$
\begin{aligned}
&= {\left[-(\lambda+\mu+n \xi) \boldsymbol{P} \boldsymbol{C}_{n+1,1}\left(t+\frac{1}{2} \Delta t\right)+\mu \boldsymbol{P} \boldsymbol{C}_{n+2,1}\left(t+\frac{1}{2} \Delta t\right)+\lambda \boldsymbol{P} \boldsymbol{C}_{n, 1}(t+\right.} \\
&\left.\left.\frac{1}{2} \Delta t\right)+(n+1) \xi\left[p_{01} \boldsymbol{P} \boldsymbol{C}_{n+2,0}\left(t+\frac{1}{2} \Delta t\right)+p_{11} \boldsymbol{P} \boldsymbol{C}_{n+2,1}\left(t+\frac{1}{2} \Delta t\right)\right]\right] \Delta t ; \\
& 1<n \leq N, k=1
\end{aligned}
$$

or 
International Journal of Mathematical, Engineering and Management Sciences

Vol. 5, No. 5, 886-896, 2020

https://doi.org/10.33889/IJMEMS.2020.5.5.068

$$
\begin{aligned}
= & {\left[-(\mu+N \xi) \boldsymbol{P} \boldsymbol{C}_{N+1,0}\left(t+\frac{1}{2} \Delta t\right)+\lambda \boldsymbol{P} \boldsymbol{C}_{N, 0}\left(t+\frac{1}{2} \Delta t\right)+N \xi\left[p_{00} \boldsymbol{P} \boldsymbol{C}_{N+1,0}(t+\right.\right.} \\
& \left.\left.\left.\frac{1}{2} \Delta t\right)+p_{10} \boldsymbol{P} \boldsymbol{C}_{N+1,1}\left(t+\frac{1}{2} \Delta t\right)\right]\right] \Delta t ; n=N+1, k=0
\end{aligned}
$$

or

$$
=\left[-(\mu+N \xi) \boldsymbol{P} \boldsymbol{C}_{N+1,1}\left(t+\frac{1}{2} \Delta t\right)+\lambda \boldsymbol{P} \boldsymbol{C}_{N, 1}\left(t+\frac{1}{2} \Delta t\right)\right] \Delta t ; n=N+1, k=1 .
$$

Vector $\mathbf{P D}$ corresponds to quantity $\left(y+k_{3}\right)$ such that $\boldsymbol{P} \boldsymbol{D}_{n, k}(t)=\boldsymbol{P} \boldsymbol{A}_{n, k}(t)+\frac{1}{2} \boldsymbol{A} \boldsymbol{C}_{n, k}$.

Vector AD corresponds to quantity $k_{4}$, therefore,

$$
\begin{aligned}
& \boldsymbol{A} \boldsymbol{D}_{n, k}=(\Delta t) \frac{d \boldsymbol{P} \boldsymbol{D}_{n, k}(t)}{d t}=\left[-\lambda \boldsymbol{P} \boldsymbol{D}_{0,0}(t)+\mu \boldsymbol{P} \boldsymbol{D}_{1,0}(t)\right] \Delta t ; n=0, k=0 \\
& \text { or } \\
& =\left[-\lambda \boldsymbol{P} \boldsymbol{D}_{0,1}(t)+\mu \boldsymbol{P} \boldsymbol{D}_{1,1}(t)\right] \Delta t ; n=0, k=1 \\
& \text { or } \\
& =\left[-(\lambda+\mu) \boldsymbol{P} \boldsymbol{D}_{1,0}(t)+\mu \boldsymbol{P} \boldsymbol{D}_{2,0}(t)+\lambda \boldsymbol{P} \boldsymbol{D}_{0,0}(t)\right] \Delta t ; n=1, k=0 \\
& \text { or } \\
& \begin{aligned}
= & {\left[-(\lambda+\mu) \boldsymbol{P} \boldsymbol{D}_{1,1}(t)+\mu \boldsymbol{P} \boldsymbol{D}_{2,1}(t)+\lambda \boldsymbol{P} \boldsymbol{D}_{0,1}(t)+\xi\left[p_{11} \boldsymbol{P} \boldsymbol{D}_{2,1}(t)+\right.\right.} \\
& \left.\left.p_{01} \boldsymbol{P} \boldsymbol{D}_{2,0}(t)\right]\right] \Delta t ; n=1, k=1
\end{aligned} \\
& =\left[-(\lambda+\mu+n \xi) \boldsymbol{P} \boldsymbol{D}_{n+1,0}(t)+\mu \boldsymbol{P} \boldsymbol{D}_{n+2,0}(t)+\lambda \boldsymbol{P} \boldsymbol{D}_{n, 0}(t)\right. \\
& \left.+n \xi\left[p_{00} \boldsymbol{P} \boldsymbol{D}_{n+1,0}(t)+p_{10} \boldsymbol{P} \boldsymbol{D}_{n+1,1}(t)\right]\right] \Delta t ; 1<n \leq N, k=0 \\
& =\left[-(\lambda+\mu+n \xi) \boldsymbol{P} \boldsymbol{D}_{n+1,1}(t)+\mu \boldsymbol{P} \boldsymbol{D}_{n+2,1}(t)+\lambda \boldsymbol{P} \boldsymbol{D}_{n, 1}(t)\right. \\
& \left.+(n+1) \xi\left[p_{01} \boldsymbol{P} \boldsymbol{D}_{n+2,0}(t)+p_{11} \boldsymbol{P} \boldsymbol{D}_{n+2,1}(t)\right]\right] \Delta t ; 1<n \leq N, k=1 \\
& =\left[-(\mu+N \xi) \boldsymbol{P} \boldsymbol{D}_{N+1,0}(t)+\lambda \boldsymbol{P} \boldsymbol{D}_{N, 0}(t)\right. \\
& \left.+N \xi\left[p_{00} \boldsymbol{P} \boldsymbol{D}_{N+1,0}(t)+p_{10} \boldsymbol{P} \boldsymbol{D}_{N+1,1}(t)\right]\right] \Delta t ; n=N+1, k=0 \\
& =\left[-(\mu+N \xi) \boldsymbol{P} \boldsymbol{D}_{N+1,1}(t)+\lambda \boldsymbol{P} \boldsymbol{D}_{N, 1}(t)\right] \Delta t ; n=N+1, k=1 \text {. }
\end{aligned}
$$

Using the vectors $\boldsymbol{A} \boldsymbol{A}_{n, k}, \boldsymbol{A} \boldsymbol{B}_{, n, k}, \boldsymbol{A} \boldsymbol{C}_{n, k}$ and $\boldsymbol{A} \boldsymbol{D}_{n, k}$ the probabilities can be computed recursively using the equation.

$P_{n, k}(t+\Delta t)=P_{n, k}(t)+\frac{1}{6}\left(\boldsymbol{A} \boldsymbol{A}_{n, k}+2 \boldsymbol{A} \boldsymbol{B}_{n, k}+2 \boldsymbol{A} \boldsymbol{C}_{n, k}+\boldsymbol{A} \boldsymbol{D}_{n, k}\right) ; n=0,1,2, \ldots N+1, k=0,1$.

\section{Numerical Illustration}

Now we obtain the time-dependent probabilities of the model by using the Runge-Kutta Method as developed above. We take $\lambda=1.8, \mu=2.5, p_{00}=0.8, p_{01}=0.2, p_{10}=0.7, p_{11}=0.3, \xi=$ 0.1 , and $N=2$. The system starts at time $t=0$ with initial condition $P_{0,0}(0)=1$. Here, we obtain the state probabilities for time $t=0.1$ as under: 


$$
\begin{aligned}
Q_{0,0}(0.1)= & Q_{0,0}(0)+\frac{1}{6}\left(\boldsymbol{A A}_{0,0}+2 \boldsymbol{A} \boldsymbol{B}_{0,0}+2 \boldsymbol{A} \boldsymbol{C}_{0,0}+\boldsymbol{A} \boldsymbol{D}_{0,0}\right)=0+\frac{1}{6}[0.25+ \\
& 2(0.17375)+2(0.19779375)+0.1302379425]=0.1872209070, \\
Q_{0,1}(0.1)= & Q_{0,0}(0)+\frac{1}{6}\left(\boldsymbol{A} \boldsymbol{A}_{0,1}+2 \boldsymbol{A} \boldsymbol{B}_{0,1}+2 \boldsymbol{A} \boldsymbol{C}_{0,1}+\boldsymbol{A} \boldsymbol{D}_{0,1}\right)=0+\frac{1}{6}[0+2 * 0+2 * \\
& 0.0000225+0.00001593]=0.00001155, \\
P_{0,0}(0.1)= & P_{0,0}(0)+\frac{1}{6}\left(\boldsymbol{A} \boldsymbol{A}_{1,0}+2 \boldsymbol{A} \boldsymbol{B}_{1,0}+2 \boldsymbol{A} \boldsymbol{C}_{1,0}+\boldsymbol{A} \boldsymbol{D}_{1,0}\right)=1+\frac{1}{6}(-0.43+ \\
2 & 2(-0.29255)+2(-0.33663675)+0.2153323725)=0.682715681, \\
P_{0,1}(0.1)= & P_{0,1}(0)+\frac{1}{6}\left(\boldsymbol{A} \boldsymbol{A}_{1,1}+2 \boldsymbol{A} \boldsymbol{B}_{1,1}+2 \boldsymbol{A} \boldsymbol{C}_{1,1}+\boldsymbol{A} \boldsymbol{D}_{1,1}\right)=0+\frac{1}{6}(0+2 * \\
& 0.00018+2 * 0.00007992+0.0002482706)=0.0001280117, \\
P_{1,0}(0.1)= & P_{1,0}(0)+\frac{1}{6}\left(\boldsymbol{A} \boldsymbol{A}_{2,0}+2 \boldsymbol{A} \boldsymbol{B}_{2,0}+2 \boldsymbol{A} \boldsymbol{C}_{2,0}+\boldsymbol{A} \boldsymbol{D}_{2,0}\right)=0+\frac{1}{6}(0.18+2 * \\
& 0.1182+2 * 0.1387438+0.0848200608)=0.1299181368, \\
P_{1,1}(0.1)= & P_{1,1}(0)+\frac{1}{6}\left(\boldsymbol{A} \boldsymbol{A}_{2,1}+2 \boldsymbol{A} \boldsymbol{B}_{2,1}+2 \boldsymbol{A} \boldsymbol{C}_{2,1}+\boldsymbol{A} \boldsymbol{D}_{2,1}\right)=0+\frac{1}{6}(0+2 * 0+2 * \\
& 0.0000162+0.0000101736)=0.0000070956 .
\end{aligned}
$$

Sum of probabilities: $Q_{0,0}(0.1)+Q_{0,1}(0.1)+P_{0,0}(0.1)+P_{0,1}(0.1)+P_{1,0}(0.1)+P_{1,1}(0.1)=$ 1.000001382. Thus, we have obtained all the time-dependent probabilities of the model by applying Runge-Kutta Method.

To validate our model, we use the "ode45" function of MATLAB software to obtain the transient numerical results for different values of parameters.

\section{Performance Measures}

We study the following performance measures:

(i) Expected system size $L_{s}(t)=\sum_{n=0}^{N}(n+1)\left[P_{n, 0}(t)+P_{n, 1}(t)\right]$.

(ii) Expected waiting time in the system $W_{S}(t)=\frac{L_{S}(t)}{\mu\left[1-Q_{0,0}(t)-Q_{0,1}(t)\right]}$.

The variations in these performance measures with respect to time are shown in figures 2 and 3 . Figure 2 shows the variation in expected system size $\left(L_{s}(t)\right)$ with time. It can be seen that that the expected system size increases with time to some extent and then asymptotically reaches the steady-state. Similarly, in figure 3, the variation in expected waiting time of a customer in the system $\left(W_{s}(t)\right)$ is shown. The waiting time also increases with time, and then asymptotically reaches the steady-state. The values of the parameters are taken as: $\lambda=5.2, \mu=6, \xi=$ $0.3, N=20, p_{00}=0.8, p_{01}=0.2, p_{10}=0.7$ and $p_{11}=0.3$. 
International Journal of Mathematical, Engineering and Management Sciences

Vol. 5, No. 5, 886-896, 2020

https://doi.org/10.33889/IJMEMS.2020.5.5.068

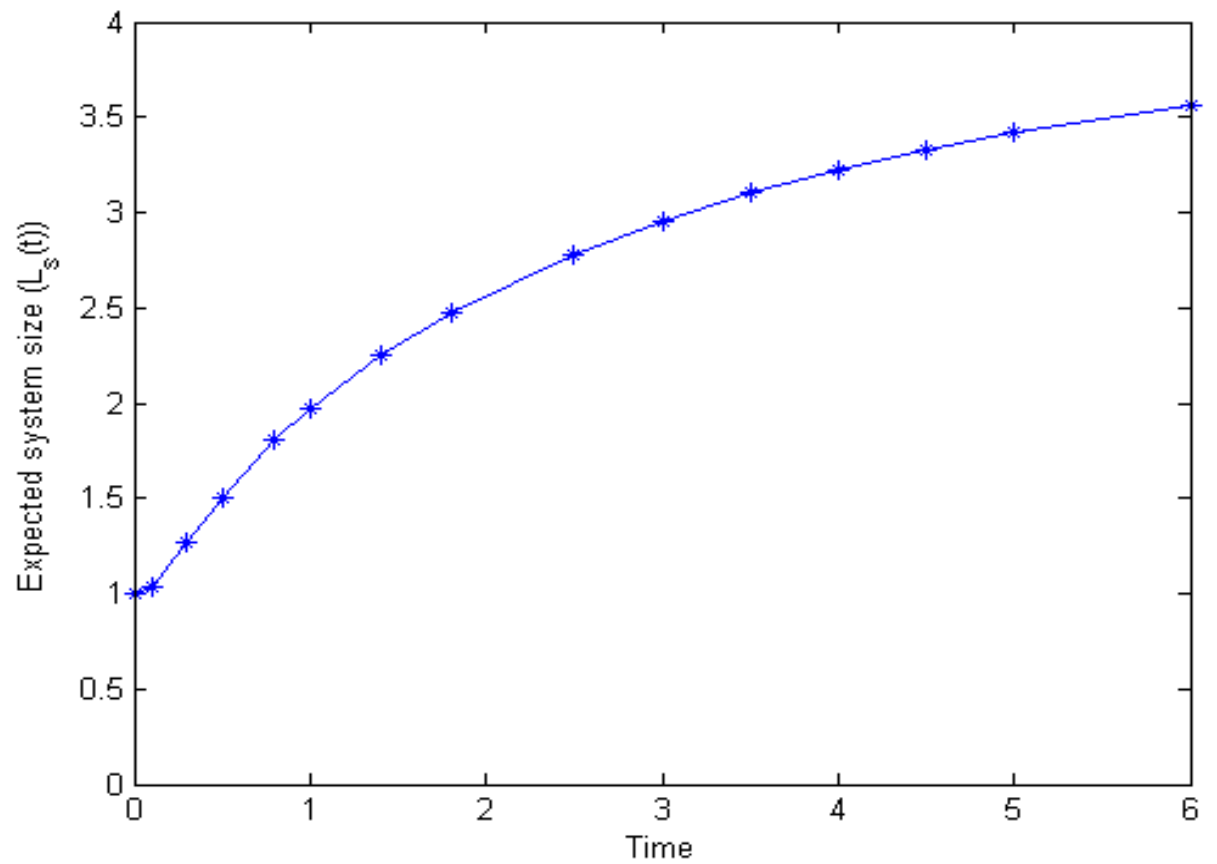

Figure 2. Expected system size vs. time

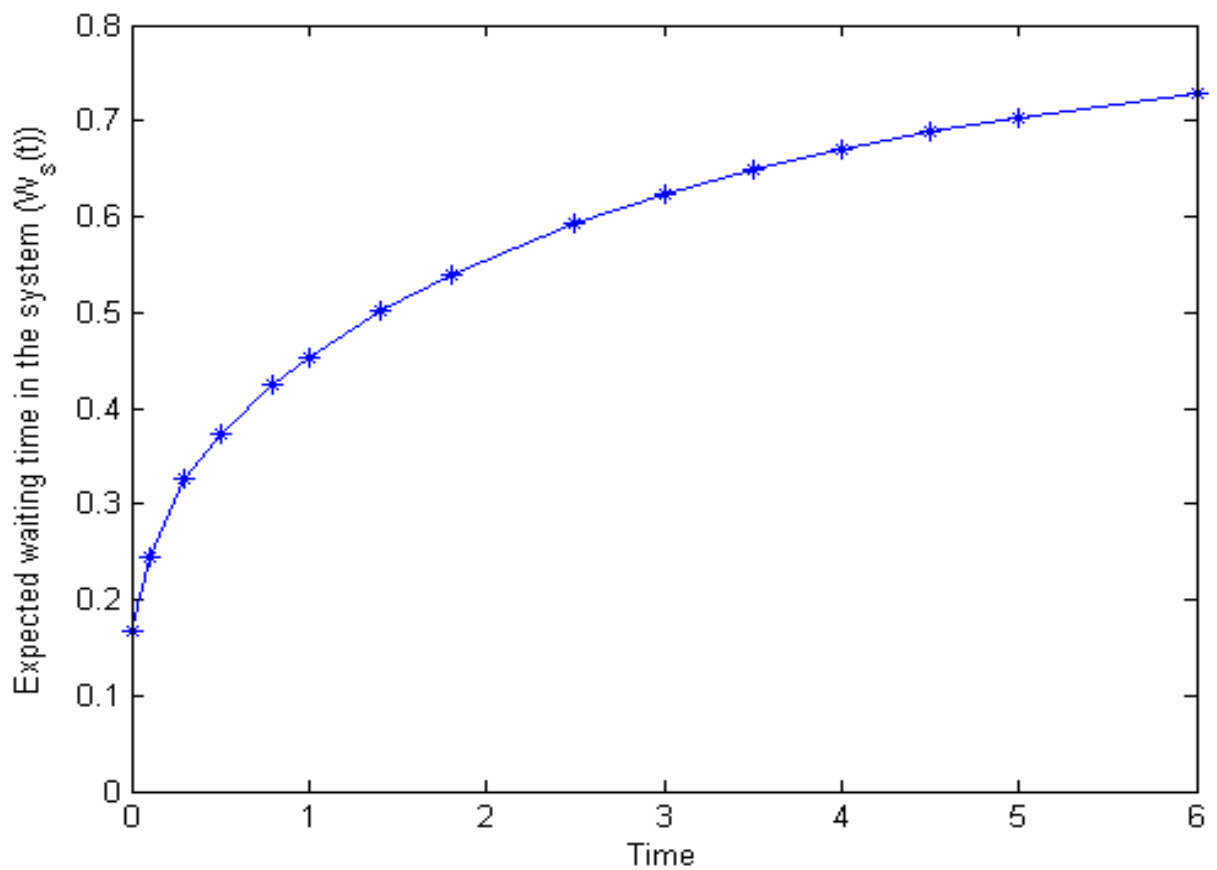

Figure 3. Expected waiting time in the system vs. Time 
International Journal of Mathematical, Engineering and Management Sciences

Vol. 5, No. 5, 886-896, 2020

https://doi.org/10.33889/IJMEMS.2020.5.5.068

\section{Conclusions and Future Work}

In this paper, the concept of correlated reneging has been introduced in queuing theory. A single server, finite capacity queuing system with correlated reneging is studied. The transient solution of the model is obtained using Runge-Kutta Method. A numerical example is provided to illustrate the functioning of the model.

In future, the model can be extended to include the effect of balking. The same work can be extended to include generally distributed inter-arrival and service times. The multi-server analogue of the model can also be obtained. Same model can be extended where arrivals are also correlated in nature.

\section{Conflict of Interest}

The authors confirm that there is no conflict of interest to declare for this publication.

\section{Acknowledgements}

The authors would like to thank the editor-in-chief and the anonymous referees for their comments and suggestions which helped us to bring this manuscript in the current form.

\section{References}

Ancker Jr, C.J., \& Gafarian, A.V. (1963a). Some queuing problems with balking and reneging. I. Operations Research, 11(1), 88-100.

Ancker Jr, C.J., \& Gafarian, A.V. (1963b). Some queuing problems with balking and reneging-II. Operations Research, 11(6), 928-937.

Cidon, I., Guérin, R., Khamisy, A., \& Sidi, M. (1993). Analysis of a correlated queue in a communication system. IEEE Transactions on Information Theory, 39(2), 456-465.

Conolly, B.W. (1968). The waiting time process for a certain correlated queue. Operations Research, 16(5), 1006-1015.

Conolly, B.W., \& Hadidi, N. (1969). A correlated queue. Journal of Applied Probability, 6(1), 122-136.

De Clercq, S., \& Walraevens, J. (2020). Delay analysis of a two-class priority queue with external arrivals and correlated arrivals from another node. Annals of Operations Research, 1-16. DOI: 10.1007/s10479-020-03548-1.

Fiems, D., \& de Turck, K. (2019). Taylor-series approximations for queues with arrival correlation. Applied Mathematical Modelling, 69, 113-126.

Haight, F.A. (1959). Queueing with reneging. Metrika, 2(1), 186-197.

Haight, F.A. (1957). Queuing with Balking. Biometrika, 44(3/4), 362-369.

Kumar, R., \& Sharma, S.K. (2012). M/M/1/N queuing system with retention of reneged customers. Pakistan Journal of Statistics and Operation Research, 8(4), 859-866.

Kumar, R., \& Sharma, S. (2018). Transient performance analysis of a single server queuing model with retention of reneging customers. Yugoslav Journal of Operations Research, 28(3), 315-331. 
International Journal of Mathematical, Engineering and Management Sciences

Vol. 5, No. 5, 886-896, 2020

https://doi.org/10.33889/IJMEMS.2020.5.5.068

Kumar, R., \& Sharma, S. (2019). Transient solution of a two-heterogeneous servers' queuing system with retention of reneging customers. Bulletin of the Malaysian Mathematical Sciences Society, 42(1), 223240.

Kumar, R., \& Soodan, B.S. (2019). Transient analysis of a single-server queuing system with correlated inputs and reneging. Reliability: Theory \& Applications, 14(1), 102-106.

Mohan, C. (1955). The gambler's ruin problem with correlation. Biometrika, 42(3/4), 486-493.

Mohan, C., \& Murari, K. (1972). Time dependent solution of a correlated queueing problem with variable capacity. Metrika, 19(1), 209-215.

Murari, K. (1969). A queueing problem with correlated arrivals and general service time distribution. ZAMM-Journal of Applied Mathematics and Mechanics/Zeitschrift für Angewandte Mathematik und Mechanik, 49(3), 151-156.

Rao, S.S. (1965). Queuing models with balking, reneging, and interruptions. Operations Research, 13(4), 596-608.

Rao, S.S. (1967). Queuing with balking and reneging in M| G| 1 systems. Metrika, 12(1), 173-188.

Sharma, S., Kumar, R., \& Ammar, S.I. (2019). Transient and steady-state analysis of a queuing system having customers' impatience with threshold. RAIRO-Operations Research, 53(5), 1861-1876.

Sudhesh, R., \& Azhagappan, A. (2019). Analysis of an M/M/C queue with heterogeneous servers, balking and reneging. International Journal of Operational Research, 36(3), 293-309.

Vishnevskii, V.M., \& Dudin, A.N. (2017). Queueing systems with correlated arrival flows and their applications to modeling telecommunication networks. Automation and Remote Control, 78(8), 13611403. 